\title{
Reliability assessment of stress concentration performance state for a perforated composite plate under traction
}

\author{
S. Attajkani ${ }^{1}$, A. Khamlichi ${ }^{1}$, A. Jabbouri ${ }^{2}$ \\ ${ }^{1}$ Department of Physics, FS Tetouan, Morocco \\ ${ }^{2}$ MGC Laboratory, FST Tangier, Morocco
}

\begin{abstract}
Considering a perforated sandwich plate made from two elastic homogenous and isotropic layers, and having a square hole, reliability assessment of stress concentration limit state for which the stress should not exceed a given threshold is performed in this work. Assuming that the plate dimensions and the applied loading are deterministic, focus is done on the square hole centre position and edge length considered to be random variables. The means and the standard deviations of these variables are assumed to be known, but no information is so far available about their densities of probabilities. To assess reliability of the performance state, reliability analysis known methods are applied to a response surface representation of the stress concentration factor of the perforated plate which is obtained through quadratic polynomial regression of finite element results. A parametric study is performed regarding the influence of the distributions of probabilities chosen to model the hole dimensions uncertainties. It is shown that the probability of failure depends largely on the selected densities of probabilities.
\end{abstract}

\section{Introduction}

Reliability is associated to the ability of a system to accomplish some required functions, during a considered period of time [1]. Reliability concepts set safety of structures within the context where there is no perfect knowledge about the real data intervening in the problem but where statistics that describe their variations are available [2,3]. For a given performance state of a structural system, it is then possible to take into account uncertainties that affect mechanical, geometrical and loading parameters. This is performed by modelling uncertainties propagation between the inputs consisting of the basic variables and the desired system outputs which define the considered performance states.

Most of structures and engineering components have perforated holes of various geometries. It is well recognized that these cause locally high stresses and act hence as stress raisers. If the components are subjected to cyclic loading for which the material cannot relieve such high stresses, they could suffer a sudden fracture that happens more promptly in comparison with unnotched structures. The presence of perforated holes or notches can reduce dramatically the fatigue life of components in some circumstances [4,5]. Many studies have dealt with the effect of holes of various shapes and dimensions on fatigue life of engineering components under cyclic loading $[5,6]$. In the case of cut-outs and holes, edge bluntness and rotation were recognized to be significant design variables and a number of works have dealt with these effects on composite plates [7,8].

In real structures, cut-outs and holes have features that are not intentional in their initial design. They constitute what it is called geometric tolerances. These can have non negligible influence on the considered limit states and act as additional stress raisers. In this work, First Order Reliability Method (FORM) and Second Order Reliability Method (SORM) are applied to a response surface representation of the stress concentration factor associated to the perforated plate subjected to traction loading. The response surface is derived through quadratic polynomial regression of finite element simulation results that are performed according to a full factorial design of experiment. The location of the centre of the square hole on the perforated composite plate and the hole edge length are assumed to be random variables. Their means and standard deviations are supposed here to be known while their densities of probabilities functions are unknown. A parametric study is performed by means of Comrel software regarding the influence of the distributions of probabilities chosen to model the hole characteristics uncertainties on reliability results.

\section{Stress concentration factor}

Stress concentration is one of the most important factors that affect fatigue life of components and structures. Stress concentration may be intentional in the design or 
resulting from imperfections such as deep machining marks or other processing related defects.

The existence of irregularities or discontinuities in a structure, such as holes, grooves, or notches increases the magnitude of stresses significantly in the immediate vicinity of the discontinuity [9]. Fatigue failure mostly originates from such places. Usually a stress concentration factor is defined to account for stress concentration effect. This factor is used to indicate the increase in the stress. Fatigue data for an unnotched specimen are related to a stress concentration factor $\mathrm{K}_{\mathrm{t}}$ equal to 1 . The amount of stress around a notch is defined as

$$
\sigma_{\max }=\mathrm{K}_{\mathrm{t}} \sigma_{\text {nom }}
$$

where $K_{t}$ is the theoretical stress concentration factor and $\sigma_{\text {nom }}$ is the nominal stress.

The presence of a notch reduces the allowable nominal stress amplitude at any fatigue life by a factor which could be very close to $\mathrm{K}_{\mathrm{t}}$ for some materials that are very sensitive to stress concentration.

In practice, fatigue data for perforated and notched elements is variable. The variability that affects life of these components has various origins. In this work, only the part due to geometric tolerances is considered through a reliability based analysis. The hole of the perforated plate is assumed to be affected by geometric variations that change its ideal characteristics according to continuous random variables. This uncertainty is propagated and its effect quantified on the value of $\mathrm{K}_{\mathrm{t}}$.

\section{Reliability analysis}

Structures are subject to inherent uncertainties due to geometric variations, stochastic material properties and applied loading. Reliability of structures represents the relevant tool which enables to calculate the probability of failure starting from the densities of probabilities of the random variables which are present in the problem, [10]. In some problems, the performance function (limit state) is not given explicitly. Coupling finite element software with a reliability code can then be used to perform reliability assessment of a given design state. But, this process is long and not all the time robust as it might fail to converge in the presence of strong nonlinearities.

An alternative way of performing reliability analysis consists in approximating the implicit performance function. These methods yield a simplified explicit mathematical representation of the exact limit state. Its evaluation in terms of the random variables that are present as inputs of the process is then straightforward. This permits to avoid systematic calls for finite element computations that are usually needed within the context of the coupled approach in order to evaluate the performance function associated to a given set of parameters, [11]. Call for the finite element code is thus managed more efficiently in order to get maximum information without increasing excessively the computational cost.

The explicit representation model is constructed by performing pertinent trial of points over the investigated domain of basic variables. The common approach uses the points that respond to a full factorial table built on a particular discrete set of factors [11]. Using the results of simulations for these points, a metamodel can be derived such that the state function is obtained as a simple analytical expression over the entire domain used for regression; such a model is called a response surface model (RSM). This takes usually the form of a polynomial function. The obtained approximation for the failure function by means of regression techniques is however valid only on the investigated domain and its extrapolation outside of it is not a priori appropriate.

In this work, quadratic regression for RSM derivation and reliability analysis software Comrel [12] are used.

Comrel offers two modules for time invariant and time variant reliability analysis of failure modes based on FORM/SORM methodology of Monte Carlo process. Several algorithms to find the most likely failure point ( $\beta$-point) are implemented. Additional computational options available in this software include Monte Carlo adaptive sampling and several importance sampling schemes. Comrel can deal with arbitrary dependence structures in the stochastic model. A full set of 44 stochastic models are available in the actual version Comrel V.7 and can be entered either in parameter form or in terms of the first two moments with additional parameters. New user defined models can also be added.

In Comrel several failure criteria can be considered simultaneously. For the symbolic version of Comrel software used in this study, the state functions can be specified in normal mathematical notation. A number of built-in functions are pre-defined. These include all elementary, trigonometric, hyperbolic, logarithmic and special functions like the Gaussian distribution function and its inverse, Bessel and Gamma functions.

\section{Results and discussion}

A composite plate made from two homogenous and isotropic elastic layers is considered, figure 1 . The plate has a square hole for which the centre vertical position and edge length are subject to tolerance variations that are assumed to be random and distributed according to unknown densities of probabilities. It has length $\mathrm{a}=1 \mathrm{~m}$, width $\mathrm{b}=0.5 \mathrm{~m}$ and thickness $\mathrm{e}=0.05 \mathrm{~m}$. Each layer of the plate has a thickness equal to $\mathrm{e} / 2=0.025 \mathrm{~m}$. The hole centre position is denoted $\mathrm{x}$ and its edge length $\mathrm{y}$. The composite plate is assumed to be clamped at its left edge and subjected at its right edge to a traction force. The mechanical properties are given in table 1 .

The ideal profile of the hole is square with an edge equal to $5 \times 10^{-2} \mathrm{~m}$ and perfectly centred vertically. The force traction acting on the right edge of the plate is associated to pressure $\mathrm{P}=10^{7} \mathrm{~Pa}$. The aim is to quantify the stress intensity factor giving the maximum stress as it could be 
changed by the tolerances affecting the input parameters: actual characteristics of the square hole. To perform that, a finite element modelling of the composite plate is achieved. Then the maximum stresses are extracted from the results. These are used to derive a RSM model by quadratic polynomial regression.

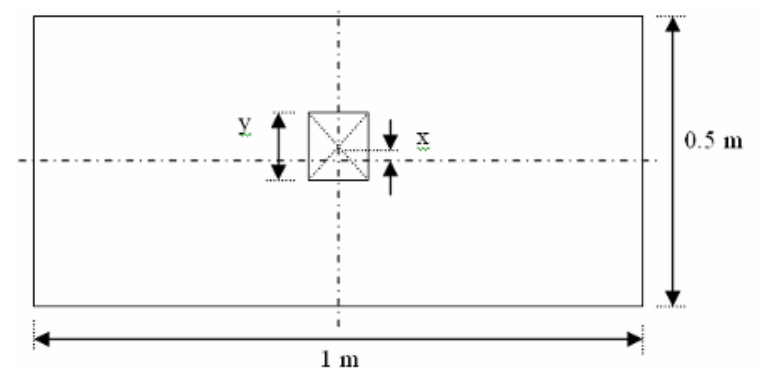

Fig.1. Plate geometric characteristics showing the presence of a square hole at approximately the centre of the plate

Table1: Material properties of the two layers composing the perforated plate

\begin{tabular}{|c|c|c|}
\hline $\begin{array}{c}\text { Layer } \\
\text { number }\end{array}$ & Young modulus $(\mathrm{Pa})$ & Poisson coefficient \\
\hline \hline 1 & $\mathrm{E}_{1}=2 \times 10^{11}$ & $\mathrm{v}_{1}=0.3$ \\
2 & $\mathrm{E}_{2}=1 \times 10^{11}$ & $\mathrm{v}_{2}=0.25$ \\
\hline
\end{tabular}

Table2: Levels of geometric characteristics of the hole as they are affected by tolerances

\begin{tabular}{|l|c|c|c|}
\hline \multicolumn{1}{|c|}{ Basic variable } & $\begin{array}{c}\text { Lower } \\
\text { value } \\
(\mathrm{m})\end{array}$ & $\begin{array}{c}\text { Intermediate } \\
\text { value }(\mathrm{m})\end{array}$ & $\begin{array}{c}\text { Higher } \\
\text { value } \\
(\mathrm{m})\end{array}$ \\
\hline $\begin{array}{l}\text { Vertical position of } \\
\text { the hole centre, } \mathrm{x}\end{array}$ & 0 & $2 \times 10^{-3}$ & $4 \times 10^{-3}$ \\
\hline $\begin{array}{l}\text { Edge length of the } \\
\text { hole, } \mathrm{y}\end{array}$ & $4.8 \times 10^{-2}$ & $5 \times 10^{-2}$ & $5.2 \times 10^{-2}$ \\
\hline
\end{tabular}

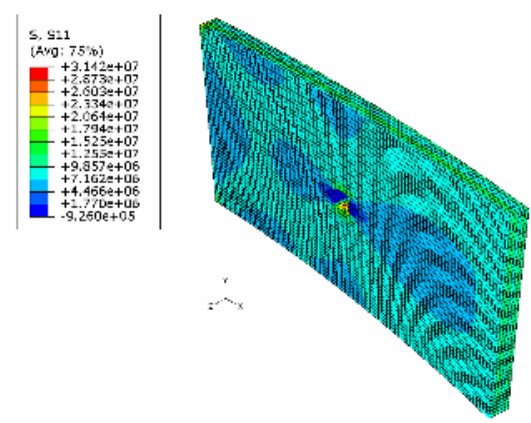

Fig.2. Stress isovalues for the combination $\mathrm{x}=4 \times 10^{-3} \mathrm{~m}$

$$
\text { and } \mathrm{y}=5.2 \times 10^{-2} \mathrm{~m}
$$

Figure 2, shows the stress isovalues obtained by means of Abaqus software. 35588 elements C3D8I (8-node linear brick with incompatible modes) were used. Convergence of the finite element method results was assessed by using the asymptotic convergence criterion. Table 3 gives the obtained results in terms of the stress concentration factor defined in equation (1) as $\mathrm{K}_{\mathrm{t}}=\sigma_{\mathrm{xx}, \max } / \sigma_{\mathrm{xx}, \mathrm{nom}}$.

\begin{tabular}{|c|c|c|}
\hline $\begin{array}{c}\text { Vertical position } \\
\text { of the hole centre, } \\
\mathrm{x}\end{array}$ & $\begin{array}{c}\text { Edge length of } \\
\text { the hole, } \mathrm{y}\end{array}$ & $\begin{array}{c}\text { Stress } \\
\text { concentration } \\
\text { factor, } \mathrm{K}_{\mathrm{t}}\end{array}$ \\
\hline \hline 0 & $4.8 \times 10^{-2}$ & 2.119 \\
0 & $5 \times 10^{-2}$ & 2.127 \\
0 & $5.2 \times 10^{-2}$ & 2.134 \\
$2 \times 10^{-3}$ & $4.8 \times 10^{-2}$ & 2.118 \\
$2 \times 10^{-3}$ & $5 \times 10^{-2}$ & 2.126 \\
$2 \times 10^{-3}$ & $5.2 \times 10^{-2}$ & 2.133 \\
$4 \times 10^{-3}$ & $4.8 \times 10^{-2}$ & 2.117 \\
$4 \times 10^{-3}$ & $5 \times 10^{-2}$ & 2.124 \\
$4 \times 10^{-3}$ & $5.2 \times 10^{-2}$ & 2.132 \\
\hline
\end{tabular}

Table 3: Stress concentration factor as function of the considered combination of the square hole characteristics,

the nominal value of stress is $\sigma_{x x, n o m}=1.575 \times 10^{7} \mathrm{~Pa}$

The RSM model as obtained by quadratic polynomial regression using results of table 3 is given by

$$
\begin{aligned}
\mathrm{K}_{\mathrm{t}}(\mathrm{x}, \mathrm{y})= & 1.8351-0.41667 \mathrm{x}+7.91667 \mathrm{y} \\
& -0.28709 \mathrm{xy}-41.667 \mathrm{x}^{2}-41.667 \mathrm{y}^{2}
\end{aligned}
$$

Let us assume that the limit state considered in design against for example fatigue loads is $\mathrm{K}_{\mathrm{t} 0}=2.125$. Then, the associated performance function writes

$\mathrm{g}(\mathrm{x}, \mathrm{y})=\mathrm{K}_{\mathrm{t} 0}-\mathrm{K}_{\mathrm{t}}(\mathrm{x}, \mathrm{y})$

Table 4 gives the results in terms of probability of failure associated to the performance state defined by equation (3).

One can notice that for low probabilities which are often the useful domain in practice, the probability of failure varies a lot when changing the densities of probabilities modelling the basic variables uncertainties. These variations start for $\mathrm{K}_{\mathrm{t} 0} \geq 1.45$. The contrast is found to be important as some variations can reach $100 \%$ of the stress concentration factor value. Table 4 gives also a comparison between the reliability analysis results obtained by FORM, SORM and crude Monte Carlo (MC) methods. Simulations have shown that FORM and SORM methods give the same probabilities of failure. But, Monte Carlo probability of failure is different from that of FORM/SORM methods when $\mathrm{K}_{\mathrm{t} 0} \geq 1.50$.

For low probabilities of failure MC method fails to give accurate predictions. To obtain that, the sample size should be excessively high. So, in practice FORM/SORM methods are more suitable in this case. Effectiveness of FORM/SORM methods is evident when moderate values of probabilities of failure are considered; no significant difference exists between these simplified reliability analysis methods and the MC 
process which is then very accurate if one uses 1000000 samples.

\begin{tabular}{|c|c|c|c|c|}
\hline $\mathrm{K}_{\mathrm{t} 0}$ & $\begin{array}{c}\text { Normal } \\
\text { (FORM/ } \\
\text { SORM) }\end{array}$ & $\begin{array}{c}\text { Normal } \\
\mathrm{MC} \\
1000000\end{array}$ & $\begin{array}{c}\text { Lognormal } \\
\text { (FORM/ } \\
\text { SORM) }\end{array}$ & $\begin{array}{c}\text { Gamma } \\
\text { (FORM/ } \\
\text { SORM) }\end{array}$ \\
\hline \hline 2.100 & 1 & 1 & 1 & 1 \\
2.105 & 1 & 1 & 1 & 1 \\
2.110 & 0.98 & 0.98 & 0.98 & 0.98 \\
2.115 & 0.92 & 0.91 & 0.93 & 0.92 \\
2.120 & 0.77 & 0.76 & 0.78 & 0.78 \\
2.125 & 0.54 & 0.52 & 0.55 & 0.55 \\
2.130 & 0.28 & 0.27 & 0.29 & 0.30 \\
2.135 & 0.10 & 0.0970 & 0.11 & 0.11 \\
2.140 & 0.0248 & 0.0223 & 0.0298 & 0.0292 \\
2.145 & $3.51 \times 10^{-3}$ & $3.01 \times 10^{-3}$ & $5.20 \times 10^{-3}$ & $4.81 \times 10^{-3}$ \\
2.150 & $2.73 \times 10^{-4}$ & $2.26 \times 10^{-4}$ & $5.81 \times 10^{-4}$ & $4.82 \times 10^{-4}$ \\
2.155 & $1.07 \times 10^{-5}$ & $1.10 \times 10^{-5}$ & $4.02 \times 10^{-5}$ & $2.81 \times 10^{-5}$ \\
2.160 & $1.92 \times 10^{-7}$ & 0 & $1.65 \times 10^{-6}$ & $8.94 \times 10^{-7}$ \\
2.165 & $1.38 \times 10^{-9}$ & 0 & $3.88 \times 10^{-8}$ & $1.46 \times 10^{-8}$ \\
2.170 & 0 & 0 & $4.87 \times 10^{-10}$ & $1.11 \times 10^{-10}$ \\
\hline
\end{tabular}

Table 4: Probability of failure as obtained for a particular choice of densities of probabilities and reliability analysis method

The results show also that Normal distributions of probabilities stop to give a significant probability of failure for $\mathrm{K}_{\mathrm{t} 0} \geq 1.70$ while Lognormal and Gamma continue to give in this case probabilities that are different from 0 .

By comparing the probabilities of failure, given in table 4 , as function of the three densities of probabilities, the Lognormal distributions give always the more conservative results. So, they can rather be used to model uncertainties in problems where the densities of probabilities are not known.

\section{Conclusion}

Reliability assessment of stress concentration limit state for a composite perforated plate with a square hole was performed in this work. The plate was modelled by using the finite element method and a response surface model that gives the stress concentration factor in terms of the centre position of the hole and its edge length was derived. The probability of failure associated to a given performance state for admissible stress concentration was computed by considering various densities of probabilities functions to model uncertainties. The obtained results have indicated that the chosen densities of probabilities to model uncertainties affecting geometric characteristics of the hole influence a lot the calculated probability of failure, mainly in the useful domain corresponding to low probabilities. The results have shown also that the Lognormal distribution of probability is the most conservative one among the three studied. So, when densities of probabilities are unknown, it is safer to use as default this distribution.

\section{References}

1. AFNOR. Eurocode 1: Bases du Calcul et Actions sur les Structures et Document d'Application Nationale. Technical Report XP ENV 1991-1, AFNOR, 1996.

2. M. Lemaire. Reliability and mechanical design. Reliability Engineering and System Safety, 55 (2): 63170, 1997.

3. A. Der Kiureghian and T. Dakessian. Multiple design points in first and second-order reliability. Structural Safety, 20(1): 37-50, 1998.

4. D.S. Um, S.W. Kang, J.H. Park, and W.I. Ha, A Study on the Stress Concentration Factor and Fatigue Strength for T-Tubular Joins by FEM, Journal of Ocean Engineering and Technology, 8 (2): 141-150, 1994.

5. G.H. Majzoobi, N. Daemi. The study of notch geometry on fatigue life using notch sensitivity factor. Transaction of the Indian Institute of Metals Journal. Vol. 63: $547-55,2010$.

6. G.N. Savin, Stress Concentration around Holes, Pergamon Press, New York, 1961.

7. W.D. Pilkey, D.F. Pilkey, R.E. Peterson, Peterson's Stress Concentration Factors, John Wiley and Sons, New York , 2008.

8. J. Woo, W.B Na. Effect of Cutout Orientation on Stress Concentration of Perforated Plates with Various Cutouts and Bluntness. International Journal of Ocean System Engineering 1(2): 95-101, 2011.

9. W.J. O'Donnel, B.F. Langer. Design of perforated plates. Journal of Engineering for Industry, Trans. ASME, vol. 84, paper No. 61-WA-115, 1962.

10. O. Ditlevsen and H.O. Madsen. Structural reliability methods, Ed. John Wiley and Sons, Hoboken, NJ, USA, 1996.

11. W.J. Roux, N. Stander and R.T. Haftka Response surface approximations for structural optimization. Journal for Numerical Methods in Engineering, 42: $517-$ $534,1998$.

12. http://www.strurel.de/comrel.htm 